\title{
The effect of high leverage points on the logistic ridge regression estimator having multicollinearity
}

\begin{abstract}
This article is concerned with the performance of logistic ridge regression estimation technique in the presence of multicollinearity and high leverage points. In logistic regression, multicollinearity exists among predictors and in the information matrix. The maximum likelihood estimator suffers a huge setback in the presence of multicollinearity which cause regression estimates to have unduly large standard errors. To remedy this problem, a logistic ridge regression estimator is put forward. It is evident that the logistic ridge regression estimator outperforms the maximum likelihood approach for handling multicollinearity. The effect of high leverage points are then investigated on the performance of the logistic ridge regression estimator through real data set and simulation study. The findings signify that logistic ridge regression estimator fails to provide better parameter estimates in the presence of both high leverage points and multicollinearity.
\end{abstract}

Keyword: High leverage points; Logistic ridge regression; Maximum likelihood; Multicollinearity 